\title{
Observational cross-sectional study of Trichomonas tenax in patients with periodontal disease attending a Chilean university dental clinic
}

Casandra Bracamonte-Wolf ${ }^{1}$, Patricio R. Orrego ${ }^{2}$, Christian Muñoz ${ }^{1}$, Daniel Herrera', Joel Bravo ${ }^{3}$, Jorge Gonzalez ${ }^{1}$ Héctor Varela ${ }^{4}$, Alejandro Catalán ${ }^{1}$ and Jorge E. Araya ${ }^{1 *}$ D

\begin{abstract}
Background: The oral flagellated protozoan Trichomonas tenax has been associated with patients with periodontal disease. However, no recent studies have been conducted on the prevalence of $T$. tenax in Chile. The aim of this study was to determine the presence of $T$. tenax in patients with periodontal disease, admitted to the Dental Clinic of the University of Antofagasta, Chile, through Polymerase Chain Reaction (PCR) amplification of the beta-tubulin gene.

Methods: An observational, cross-sectional study was conducted on 50 patients diagnosed with periodontal disease, 20 of them with gingivitis and 30 with periodontitis. T. tenax was identified by PCR amplification of the beta-tubulin gene. Associations between the protozoan and periodontal disease or the presence of risk factors to establish T. tenax infection were determined using the chi-square test and binary logistic regression analysis.

Results: T. tenax was present in 28 out of $50(56 \%)$ of patients with periodontal disease, and was more prevalent when associated with periodontitis (21 out of 30; 70\%) than dental plaque-induced gingivitis (7 out of 20; 35\%). Non-statistically-significant associations were observed between the presence of $T$. tenax and age, gender, smoking habit or diabetes. Statistically significant associations were observed between the presence of T. tenax and periodontal disease, and between T. tenax and the Periodontal Screening and Recording (PSR) index.

Conclusion: T. tenax showed a high presence in patients with progressive states of periodontal diseases.

Consequently, T. tenax detection is strongly recommended in patients with periodontal disease diagnosis and with a PSR index greater than 3.
\end{abstract}

Keywords: Trichomonas tenax, PCR, Periodontal disease, Periodontitis, Gingivitis

\section{Background}

Trichomonas tenax is a flagellated, aerotolerant protozoan that lives in the human oral cavity and is distributed between the teeth, gums, tongue and saliva of people with poor oral hygiene [1]. The presence of this protozoan is considerably high in persons with more dental calculus, coated tongue and poorly-cleaned

\footnotetext{
* Correspondence: jorge.araya@uantof.cl

${ }^{1}$ Laboratory of Molecular Parasitology, Department of Medical Technology, Faculty of Health Sciences, University of Antofagasta, Angamos Avenue 601 P.O. Box 170, Antofagasta, Chile

Full list of author information is available at the end of the article
}

periodontal tissue rather than in individuals with clean and healthy oral cavities [2]. T. tenax has been implicated in the aetiology of several infections outside of the oral cavity, being detected in cerebrospinal fluid samples from patients diagnosed with polymicrobial meningitis [3], in the salivary duct infecting the sub-maxillary gland [4], and causing pulmonary eosinophilia in bronchoalveolar fluid [5]. T. tenax has also been identified in fibrocystic mastopathy [6], in a infra-auricular lymph nodes causing cervical adenopathy [7], in sputum samples of immunocompromised patients with chest pain and chronic lung diseases causing pulmonary trichomoniasis

(c) The Author(s). 2019 Open Access This article is distributed under the terms of the Creative Commons Attribution 4.0 International License (http://creativecommons.org/licenses/by/4.0/), which permits unrestricted use, distribution, and reproduction in any medium, provided you give appropriate credit to the original author(s) and the source, provide a link to the Creative Commons license, and indicate if changes were made. The Creative Commons Public Domain Dedication waiver (http://creativecommons.org/publicdomain/zero/1.0/) applies to the data made available in this article, unless otherwise stated. 
[8], and in pleural empyema samples from upper respiratory tract infections [9]. Although T. tenax has been detected in dental calculus and subgingival dental plaque, its role in the physiopathology and the mechanism involved on tissue damage of oral infections are still unclear [10]. In addition, T. tenax presence in the oral cavity has been associated with periodontal disease [11]; however, its role in this pathology is also unclear.

Periodontal disease occurs when the complex composition and organization of the periodontium is affected by a homeostatic interruption between the oral microbiome and the host, thus leading to the development of gingivitis and periodontitis, two related diseases that differ in their degree of periodontium commitment [12, 13]. Dental plaque-induced gingivitis (DPIG) is an inflammatory alteration of the soft tissue surrounding the teeth and gums, resulting from bacterial plaque accumulating on the teeth; it is clinically characterized by reddened and inflamed gums and increased gum bleeding after soft probing, which is reversible once bacterial plaque is eliminated by effective mechanical oral hygiene [14]. Gingivitis can also be modified by several factors, such as tobacco, drugs and hormonal changes occurring during puberty and pregnancy [15]. Periodontitis is considered as a progression of gingivitis, traditionally caused by bacterial plaque and characterized mostly by irreversible destruction of the supporting tissues around the teeth, periodontal ligaments, bone and soft tissue [16]. Clinically, during periodontitis, the epithelium migrates along the radicular surface, with insertion loss, increased pocket depth, and bone crest loss, which can lead to tooth loss $[15,16]$. Therefore, periodontitis is the most severe and important kind of the periodontal diseases.

T. tenax presence in periodontal diseases has been reported since the 1960s, with research interest increasing from the 1980s to the present [11]. Thus, several reports about $T$. tenax prevalence support the idea that the parasite prevalence in oral infections was much greater in patients with periodontal diseases when compared with patients with periodontal oral health $[2,11]$. In most such reports, T. tenax was detected from bacterial plaque samples by direct microscopy or culture, with prevalences that varied significantly $(0-94 \%)$ [11]. The wide range in estimated prevalences likely reflect that $T$. tenax trophozoites are highly sensitive to environmental changes, such as temperature and $\mathrm{pH}$ affecting their shape and flagella size, which make the culture and maintenance of trophozoite morphology and viability, required for subsequent microscopic identification, more difficult [1]. Hence, detecting protozoan by a more sensitive technique, such as polymerase chain reaction (PCR), could greatly improve the estimation of $T$. tenax prevalence in patients with periodontal disease. However, from our understanding only four prevalence studies have used PCR to detect this protozoan [17-20]. In Chile, the knowledge of $T$. tenax prevalence in patients with periodontal disease is poorly known, with a single report on prevalence from 1978, which showed a prevalence $38 \%$, using microscopic detection of T. tenax trophozoites for their identification [21].

In the present study, PCR was used to evaluate the presence of T. tenax in patients with periodontal disease, in order to update the prevalence data for this protozoan and to analyse its association in the development of periodontal disease and the presence of risk factors for establishing the parasite infection.

\section{Methods}

\section{Patients and samples}

We performed an observational, and cross-sectional study from May 2013 to November 2014, on people ranging from 20 to 80 years old, who were attended at the Periodontics Post-Graduate Clinic of the University of Antofagasta, Antofagasta, Chile. Inclusion criteria were the clinical diagnosis of periodontal disease. Exclusion criteria for patients were the followed: 1) not having received periodontal treatment during the last year, 2) not having taken antibiotics treatment during the last 6 months, and 3) not having dental implants. Following this, 50 patients were selected based on their periodontal diagnosis and they were classified initially according to the criteria of the American Academy of Periodontology (AAP) [22], and re-classified according to the recently guidelines to classification of periodontal disease [23] into dental plaque-induced gingivitis (DPIG) or periodontitis. Thirty patients had generalized periodontitis, where stage ranged from I to III, and grade ranged from A to B; and 20 patients had DPIG. For both groups, dental plaque and dental calculus samples were taken prior to the initiation of periodontal therapy. All patients included in the study gave their informed consent, which was approved by the Research Ethics Committee of the University of Antofagasta (CEIC-REV/2014).

Samples of dental plaque and dental calculus from patients with periodontitis were obtained from the periodontal pocket with a probing depth $\geq 5 \mathrm{~mm}$. Dental plaque samples were obtained through a gutta-percha cone, previously disinfected with $0.12 \%$ chlorhexidine, which was introduced subgingivally and rubbed for $5 \mathrm{~s}$ on the contaminated tooth surface; meanwhile, the dental calculus samples were obtained using a sterile curette. Both samples from each patient were deposited and dispersed into an independent microtube containing 1.5 $\mathrm{mL}$ sterile Ringer's solution. Moreover, samples of dental plaque and dental calculus from patients with DPIG were obtained with a sterile curette, deposited and dispersed into a microtube containing $1.5 \mathrm{~mL}$ of sterile Ringer's solution. All samples were transported to the 
Molecular Parasitology Laboratory at the University of Antofagasta within $2 \mathrm{~h}$ of collection and immediately processed to maintain protozoan viability to avoid the trophozoites lysis.

\section{Clinical registry data}

Demographic antecedents, such as gender and age, and risk factors such as diabetes status and smoking habits, were registered from clinical records of patients diagnosed with periodontitis or DPIG. Furthermore, the Periodontal Screening and Recording (PSR) index was recorded, which consists of an examination of six sites per tooth for all the patient's teeth, where the end of a periodontal WHO probe [24] was inserted gently between the tooth and gum to the depth of the dental groove. The probing depth was read by observing the position of the black band on the probe, granting each sextant a PSR index (between 0 to 4 ) and registering the highest code per sextant [25]. Similarly, the Gingival Index (GI) was recorded, following the classification criteria (between 0 and 3) described by Löe et al. [26]. Also, clinical images of each patient's teeth were recorded to document the periodontal status.

\section{Direct observation}

All samples in Ringer's solution were centrifuged at 800 $\mathrm{x} \mathrm{g}$ for $10 \mathrm{~min}$ at $20-22^{\circ} \mathrm{C}$. The supernatant was discard and the pellet was resuspended in $500 \mu \mathrm{L}$ of sterile PBS $\mathrm{pH}$ 7.2. Drops of each resuspended pellet were placed on microscope slides and examined under optic microscopy. The identification of $T$. tenax was established according to movement criteria (circular movement) under dry 400x magnification, and according to morphological criteria (pear-shaped flagellated trophozoite, about $5-13 \mu \mathrm{m}$ long) in samples stained with Giemsa and examined under 1000x immersion magnification. The remaining sample was used for DNA extraction.

\section{Genomic DNA extraction}

Genomic DNA was extracted from dental plaque and/or dental calculus samples using the inorganic method of phenol-chloroform [27]. Briefly, sediment samples were re-centrifuged at $800 \mathrm{x} \mathrm{g}$ for $10 \mathrm{~min}$ at $20-22^{\circ} \mathrm{C}$, and the pellet was lysed with $500 \mu \mathrm{L}$ of lysis buffer $(50 \mathrm{mM}$ Tris$\mathrm{HCl}, \mathrm{pH} 8.0$, 4\% Triton X-100, 62.5 mM EDTA pH 8.0, and $2.5 \mathrm{M} \mathrm{LiCl}$ ) with $1 \mathrm{mg} / \mathrm{mL}$ of RNAse A (Thermo Fisher Scientific Inc., Waltham, MA, USA), incubated for $30 \mathrm{~min}$ at $37^{\circ} \mathrm{C}$, and then incubated for $2 \mathrm{~h}$ at $50^{\circ} \mathrm{C}$ with $100 \mu \mathrm{g} / \mathrm{mL}$ of proteinase K (US Biological Life Sciences, Salem, MA, USA). Next, an equal volume of phenolchloroform-isoamyl alcohol (25:24:1) was added. The suspension was vigorously stirred and centrifuged at $13,000 \mathrm{x}$ $\mathrm{g}$ for $10 \mathrm{~min}$ at $4{ }^{\circ} \mathrm{C}$. The aqueous phase was collected and transferred into a new tube, then an equal volume of chloroform was added and centrifuged at $13,000 \mathrm{x} \mathrm{g}$ for $10 \mathrm{~min}$ at $4{ }^{\circ} \mathrm{C}$. The aqueous phase was recovered and transferred into a new tube. The DNA sample was precipitated with $0.3 \mathrm{M}$ sodium acetate at $\mathrm{pH} 5.5$ and a $2.5 \mathrm{vol}$ umes of cold absolute ethanol. The sample was centrifuged at $13,000 \times \mathrm{g}$ for $20 \mathrm{~min}$ at $4{ }^{\circ} \mathrm{C}$. The supernatant was discarded, and the precipitate was washed with $500 \mu \mathrm{L}$ of $70 \%$ ethanol, then centrifuged at $13,000 \times \mathrm{g}$ for $5 \mathrm{~min}$ at $4{ }^{\circ} \mathrm{C}$. Finally, the precipitate was dried and resuspended in $50 \mu \mathrm{L}$ of sterile free-nuclease water. Additionally, genomic DNA from $T$. tenax strain $\mathrm{Hs}-4: \mathrm{NIH}$ $\left(\right.$ ATCC $^{\bullet} 30207^{\mathrm{mm}}$ ) was extracted using the same method described.

\section{PCR amplification of the beta-tubulin gene}

PCR was performed to detect the T. tenax beta-tubulin gene. The primers used were designed from the DNA sequence of the Trichomonas vaginalis betatubulin gene (accession number: XM_001582993) [28] to amplify a 405-bp product. The primer sequences were Tt $\beta$-tub (sense) 5'-ATACTCTATCGTCCCATC TC-3' and Tt $\beta$-tub (antisense) 5'-GCCATCATGT TCTTGTTATCG-3'. The PCR reaction was performed in a reaction volume of $50 \mu \mathrm{L}$, containing: $1 \mu \mathrm{L}$ of DNA, $1 \mathrm{X}$ Taq DNA buffer $(75 \mathrm{mM}$ Tris- $\mathrm{HCl}$ $\mathrm{pH} 8.8,20 \mathrm{mM}\left(\mathrm{NH}_{4}\right)_{2} \mathrm{SO}_{4}, 0.01 \%$ (v/v) Tween 20), $0.2 \mathrm{mM}$ of each dNTP, $2.5 \mathrm{mM}$ of $\mathrm{MgCl}_{2}, 400 \mathrm{nM}$ of each primer, and $2.5 \mathrm{U}$ of Taq DNA polymerase (Thermo Fisher Scientific, Inc., Waltham, MA, USA). The reaction was performed in a T100 thermocycler (Bio-Rad), using the following reaction conditions: denaturation at $95^{\circ} \mathrm{C}$ for $3 \mathrm{~min}$, followed by 35 cycles of denaturation at $95^{\circ} \mathrm{C}$ for $30 \mathrm{~s}$, primer annealing at $46.5^{\circ} \mathrm{C}$ for $20 \mathrm{~s}$, elongation at $72^{\circ} \mathrm{C}$ for $1 \mathrm{~min}$, and a final stage of $72{ }^{\circ} \mathrm{C}$ for $7 \mathrm{~min}$. The amplification products were separated by electrophoresis in $1 \%$ agarose gel and stained with ethidium bromide (EtBr). Gel images were photo-documented using an MF-ChemiBIS 2.0 Gel Documentation System (DNR Bio-Imaging Systems, Mahale HaHamisha, Jerusalem, Israel) using Gel Capture Pro software.

\section{Statistical analysis}

Chi-square analysis was used to verify the independence hypotheses in two variables and an alternative hypothesis of association between the variables. Also, the Fisher's exact test was used for small samples. Binary logistic regression analysis was used to model the probability of detecting $T$. tenax presence based on the predictive variables, PSR and smoking habits. A statistical significance criterion level of $p<0.05$ was used. All statistical analyses were performed using Minitab v.16.0 (Minitab, LLC, State College, PA, USA). 


\section{Results}

T. tenax was observed by direct microscopy in patients with dental plaque-induced gingivitis and periodontitis (Fig. 1). Presence or absence of T. tenax in patients with periodontal disease was determined by the presence of a 405-bp PCR product for the $\beta$ tubulin gene in an agarose-gel electrophoresis (See Additional file 1: Figure S1 and Additional file 2: Figure S2). Amplification of the $\beta$-tubulin gene was also observed in samples from dental calculus and sub-gingival dental plaque from all those patients where T. tenax was recorded. From the 50 samples evaluated by PCR for T. tenax identification, 28 (56\%) showed T. tenax, of which, 12 corresponded to female patients $(24 \%)$ and 16 to male patients (32\%), with no association between patient gender and T. tenax presence $\left(\chi^{2}=2.131, p>0.05\right)$ (Table 1$)$. Examination of $T$. tenax presence by age group, showed a higher frequency of T. tenax in the 20-to 40-year-old group with 14 positive patients (50\%), followed by the 40-to 60-year-old group with 11 positive patients (34\%). No association was found between patient age and $T$. tenax presence $\left(\chi^{2}=1.811\right.$, $p>0.05$ ) (Table 1).

T. tenax frequency by periodontal diagnosis in the study population (Table 2), showed that the protozoan was present in $7 / 20$ patients (35\%) with DPIG and in $21 / 30$ patients (70\%) with periodontitis, with a statistically significant association between $T$. tenax presence and periodontal diagnosis $\left(x^{2}=5.965, p<\right.$
0.05). When analysing the association between smoking and T. tenax presence (Table 3), among the 28 patients with oral trichomoniasis, 13/28 (52\%) were smokers and 15/28 (60\%) were non-smokers, indicating no association between smoking habit and $T$. tenax presence $\left(x^{2}=0.324, p>0.05\right)$. T. tenax were only observed in $7 / 30$ diabetic patients with periodontitis $(23.3 \%)$, but not in diabetic patients with dental plaque-induced gingivitis $0 / 20(0 \%)$. A statistically significant association between diabetes and periodontal diseases was observed $\left(\chi^{2}=5.426, p=0.02\right)$. The frequency of $T$. tenax in patients with periodontitis and diabetes was 6/7 (86\%) (Table 3); however, no association between diabetes and T. tenax presence was observed (Fisher's exact test, $p=0.393$ ). With regard to the gingival index and T. tenax infection, the range in which $T$. tenax was most frequently detected was the GI between 1.1 and 2, reported from 23/50 patients, representing $46 \%$ of the cases studied. No association was found between the GI and $T$. tenax presence $\left(\mathrm{X}^{2}=1.713, p<0.05\right)$ (Table 3$)$.

Finally, relative to $T$. tenax detection and the PSR index (Table 4), our results indicated that the range in which $T$. tenax was most frequently detected was in patients with PSR index between 3.1 and 4, with 19/23 patients in this range, representing $38 \%$ of the cases studied. This indicated an association between $T$. tenax presence and the PSR index $\left(x^{2}=6.579 ; p<0.05\right)$. Additionally, logistic regression analysis for the probability of detecting T. tenax, considering the predictive variables
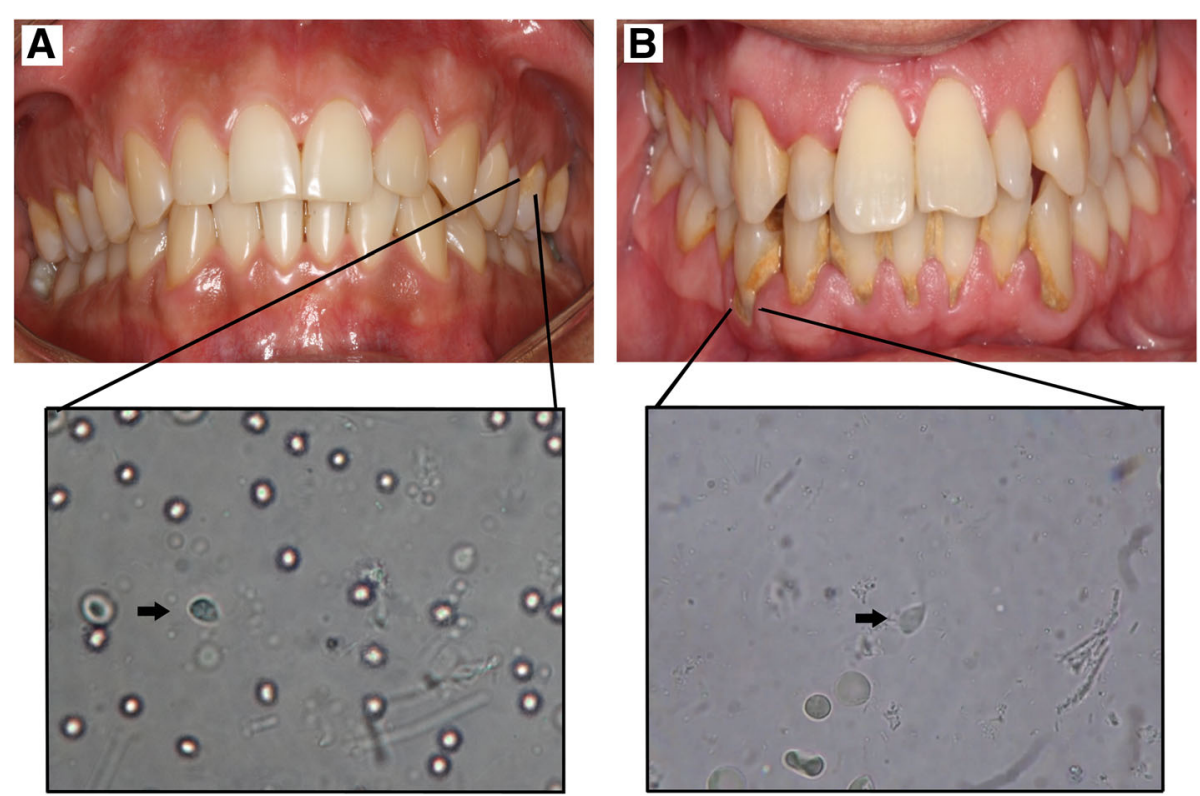

Fig. 1 Direct microscopy observation of T. tenax in patients with periodontal disease. a Representative image from a dental plaque-induced gingivitis patient. b Representative image from a periodontitis patient. Bottom: T. tenax trophozoites observed by microscopy in each patient are showed by arrows $(\times 400)$ 
Table 1 Trichomonas tenax prevalence by demographic data

\begin{tabular}{|c|c|c|c|c|}
\hline & \multirow{2}{*}{$\begin{array}{l}\text { Samples } \\
\text { (n) }\end{array}$} & \multicolumn{2}{|c|}{ Presence of $T$. tenax } & \multirow{2}{*}{$\begin{array}{l}p \\
\text { value* }\end{array}$} \\
\hline & & $\begin{array}{l}\text { YES } \\
\text { n (\%) }\end{array}$ & $\begin{array}{l}\mathrm{NO} \\
\mathrm{n}(\%)\end{array}$ & \\
\hline \multicolumn{5}{|l|}{ Gender } \\
\hline Male & 24 & $16(67)$ & $8(33)$ & \multirow[t]{3}{*}{0.144} \\
\hline Female & 26 & $12(46)$ & $14(54)$ & \\
\hline Total n (\%) & $50(100)$ & $28(56)$ & $22(44)$ & \\
\hline \multicolumn{5}{|l|}{ Age (years) } \\
\hline $20-40$ & 29 & $14(50)$ & $15(68)$ & \multirow[t]{3}{*}{0.404} \\
\hline $40-60$ & 17 & $6(27)$ & $11(39)$ & \\
\hline $60-80$ & 4 & $1(5)$ & $3(11)$ & \\
\hline Total n (\%) & $50(100)$ & $21(42)$ & $29(58)$ & \\
\hline
\end{tabular}

* Chi-square test. Significantly different $p<0.05$

PSR index and smoking, showed no association between smoking habit and T. tenax presence (binary logistic regression $p=0.127$ ). However, the probability of detecting $T$. tenax in non-smokers patients was greater than the probability of detecting the protozoan in smokers, at all PSR levels.

\section{Discussion}

For many years, it was assumed that bacteria were the only microorganisms involved in the formation of dental plaque, and they were also considered responsible for forming the dental calculus [29]. However, although largely comprised of bacteria, some fungal, mycoplasma and protozoan species are also found in dental plaque and calculus, among these the flagellated protozoan, Trichomonas tenax $[11,30]$.

Most efforts to understand the oral microbiome have focused on the bacteriological microbiota, while oral parasitology has been less studied [31]. T. tenax has recently been reported to produced damage to the mammalian epithelial cells, and it behaves similarly to $T$. vaginalis, a closely related and pathogenic Trichomonas species of the genitourinary tract, thus satisfying the requirements to be considered as a parasite [32]. Therefore, the traditional view of $T$. tenax as a commensal organism is now being questioned [32]. However, $T$.

Table 2 Trichomonas tenax infection prevalence by periodontal diagnosis

\begin{tabular}{|c|c|c|c|c|}
\hline \multirow{2}{*}{$\begin{array}{l}\text { Periodontal } \\
\text { diagnosis }\end{array}$} & \multirow{2}{*}{$\begin{array}{l}\text { Samples } \\
\text { (n) }\end{array}$} & \multicolumn{2}{|c|}{ Presence of $T$. tenax } & \multirow{2}{*}{$\begin{array}{l}p \\
\text { value* }\end{array}$} \\
\hline & & $\begin{array}{l}\text { YES } \\
\mathrm{n}(\%)\end{array}$ & $\begin{array}{l}\text { NO } \\
\text { n (\%) }\end{array}$ & \\
\hline Gingivitis & 20 & $7(35)$ & $13(65)$ & 0.0145 \\
\hline Periodontitis & 30 & $21(70)$ & $9(30)$ & \\
\hline Total n (\%) & $50(100)$ & $28(56)$ & $22(44)$ & \\
\hline
\end{tabular}

* Chi-square test. Significantly different $p<0.05$
Table 3 Trichomonas tenax prevalence by risk factors and gingival index

\begin{tabular}{|c|c|c|c|c|}
\hline \multirow[t]{2}{*}{ Variable } & \multirow{2}{*}{$\begin{array}{l}\text { Samples } \\
\text { (n) }\end{array}$} & \multicolumn{2}{|c|}{ Presence of $T$. tenax } & \multirow{2}{*}{$\begin{array}{l}p \\
\text { value* }\end{array}$} \\
\hline & & $\begin{array}{l}\text { YES } \\
\text { n (\%) }\end{array}$ & $\begin{array}{l}\mathrm{NO} \\
\mathrm{n}(\%)\end{array}$ & \\
\hline \multicolumn{5}{|c|}{ Smoking habit } \\
\hline YES & 25 & $13(52)$ & $12(48)$ & 0.568 \\
\hline NO & 25 & $15(60)$ & $10(40)$ & \\
\hline Total n (\%) & $50(100)$ & $28(56)$ & $22(44)$ & \\
\hline \multicolumn{5}{|c|}{ Diabetes mellitus ${ }^{(a)}$} \\
\hline YES & 7 & 6 & 1 & 0.300 \\
\hline NO & 23 & 15 & 8 & \\
\hline Total n (\%) & $30(100)$ & $21(70)$ & $9(30)$ & \\
\hline \multicolumn{5}{|c|}{ Gingival index } \\
\hline $0.1-1$ & 5 & 3 & 2 & 0.424 \\
\hline $1.1-2$ & 43 & 23 & 20 & \\
\hline $2.1-3$ & 2 & 2 & 0 & \\
\hline Total n (\%) & $50(100)$ & $28(56)$ & $22(44)$ & \\
\hline
\end{tabular}

${ }^{a}$ Fisher's exact test $=0.393 .{ }^{*}$ Chi-square test. Significantly different $p<0.05$

tenax's pathophysiological role in periodontal disease is unclear, since no studies in animal models have corroborated its pathological nature [11]. For these reasons, accurate identification of $T$. tenax in patients with periodontal disease is required, in order to update our understanding of prevalence and determine its association with the disease.

Currently the $T$. tenax prevalence range in patients with periodontal disease is very wide (between 0 to 94\%). This likely reflects the use of different detection methodologies, mainly comprised of conventional detection methods such as microscopic observation and cell culture, which require great operator skill and meticulous control of the conditions that allow trophozoite viability [11]. This problem could be solved by using a more sensitive and specific techniques for identification, such as PCR, which is already used to identify bacterial

Table 4 Trichomonas tenax infection prevalence by Periodontal Screening and Recording (PSR) index

\begin{tabular}{|c|c|c|c|c|}
\hline \multirow{2}{*}{$\begin{array}{l}\text { Periodontal } \\
\text { Screening } \\
\text { and } \\
\text { Recording } \\
\text { index }\end{array}$} & \multirow{2}{*}{$\begin{array}{l}\text { Samples } \\
\text { (n) }\end{array}$} & \multicolumn{2}{|c|}{ Presence of $T$. tenax } & \multirow{2}{*}{$\begin{array}{l}p \\
\text { value }^{*}\end{array}$} \\
\hline & & YES n (\%) & $\mathrm{NO} n(\%)$ & \\
\hline $1.1-2$ & 12 & $5(42)$ & 7 (58) & 0.0372 \\
\hline $2.1-3$ & 12 & $4(33)$ & $8(27)$ & \\
\hline $3.1-4$ & 26 & $19(73)$ & $7(27)$ & \\
\hline Total n (\%) & $50(100)$ & $28(56)$ & $22(44)$ & \\
\hline
\end{tabular}

* Chi-square test. Significantly different $p<0.05$ 
microorganisms in the oral cavity associated with periodontal disease [33, 34]. In this study, we used PCR with primers designed to amplify a segment of $405 \mathrm{bp}$ of the beta-tubulin gene. This allows the specific amplification of amounts as low as $100 \mathrm{fg}$ of T. tenax DNA, without interference from DNA belonging to other known pathogens of the oral cavity [35], such as Porphyromonas gingivalis, a well-known periodontal pathogen that is frequently found in patients with chronic periodontitis [29], or Aggregatibacter actinomycetemcomitans, whose colonization has been associated with aggressive periodontal disease [36]. The frequency of T. tenax detected by PCR in patients with periodontal disease was in agreement with previous studies using PCR to identify T. tenax, where the prevalence observed ranged between of 6 to $56 \%$ [17, 19, 20, 37]. A difference was also observed in $T$. tenax presence relative to periodontal disease severity (progression from dental plaque-induced gingivitis to periodontitis), being more frequently recorded in patients with periodontitis rather than in patients with gingivitis. This is likely because periodontitis involves destruction of the insertion periodontium, generating epithelial migration along the radicular surface and increasing the periodontal pocket depth, which generates anaerobic conditions that favour the establishment of the protozoan [15]. This could explain why some authors have reported higher $T$. tenax prevalence in patients with periodontitis rather than in patients with gingivitis $[18,38,39]$. However, no such differences have been reported in other studies where detection methods such as microscopy or cell culture were used [40, 41]. We demonstrated a statistically significant association between $T$. tenax presence and periodontal diagnosis: as such, screening for this protozoan needs to be considered in patients diagnosed with periodontitis.

In addition, the observational study carried on demonstrated for the first time the association between $T$. tenax presence and the PSR index. This index is based on three parameters: gingival bleeding on probing, calculus accumulation, and depth of probing, providing a detailed view of the patient's periodontal status [42]. The association between $T$. tenax presence in patients with PSR indexes $\geq 3$ allows us to consider the importance of this parameter in the screening for T. tenax infection in patients with periodontal disease. This observation is supported as deeper periodontal probing means that the periodontal environment becomes more anaerobic. This leads to a decrease in the partial pressure of oxygen, potentially explaining why the periodontal pocket depth is a critical factor for the colonization and anaerobic growth of T. tenax [11]. This observation was supported by our positive results for the presence of T. tenax in patients with PSR index of 3 or 4 and probing at depths greater than $5 \mathrm{~mm}$.
In Chile, only a single previous study examining the prevalence of $T$. tenax exists, conducted in the city of Valdivia [21]. Here, the authors used identification by optical microscopy, a reported $38 \%$ frequency $(n=50)$ of patients infected with T. tenax [21]. This frequency was lower than that reported here (56\%), likely partly because our patients had not been examined or clinically treated before, and that the authors' methodology (direct observation) was less sensitive for detecting protozoa compared to PCR.

No statistically significant association was found between gender and T. tenax presence. These results are in agreement with those obtained in other studies $[39,43]$. Some studies have reported that $T$. tenax detection is influenced by age, where its prevalence is higher in adolescents than in children [44]. It has also been reported that periodontal tissues in patients over 40 years old have greater T. tenax infections [45, 46]. However, our study did not find evidence of a significant association between age and T. tenax infection. Recently, a review of T. tenax prevalence in periodontal diseases showed that among eight studies focused on groups of children and students, the prevalence was quite low, ranging from 0 to $4 \%$, except in one study reporting a $T$. tenax prevalence of approximately $14 \%$ in young people [11]. Some authors have supported the idea that when age increases, T. tenax presence also increases $[47,48]$. However, these reports are questionable since $T$. tenax presence in the oral cavity is closely linked to the presence of teeth, such as been reported in completely edentulous patients [49] or in very young children [44], where no T. tenax were detected.

With regard to risk factors for periodontal disease, no association between $T$. tenax presence and smoking was observed. This was in accordance with previously studies, where smoking was not associated with the presence of $T$. tenax, and smoking and nonsmoking groups showed similar frequency of the protozoan [50]. However, our data for the probability of detecting $T$. tenax in non-smokers patients was greater than that for detecting the protozoa in smokers. That may be explaining because one of the first alterations in the periodontal tissues of smoking patients corresponds to epithelial hyperplasia and gingival recession [51], which are associated with vasoconstriction [52] and fewer blood vessels [53]. This causes less iron availability in the environment, which could affect $T$. tenax adherence to the gingival tissues, since studies of cytoadherence performed in the closely related species, $T$. vaginalis, indicate that adhesion levels are mediated specifically by iron [54]. Therefore, non-smokers could provide a more favourable environment for the development of the protozoan. Thus, further studies are needed with more smoking/non-smoking patients to corroborate 
the above results at experimental levels. However, the fact that smokers show reduced infection by $T$. tenax could suggest a protective effect of nicotine against infection by the parasite. Experimental studies have shown that nicotine has protective effect against pneumonia caused by Pneumocystis carinii [55]. Also, the periodontal pathogen Porphyromonas gingivalis can adapt to nicotine exposure over time and develops tolerance to the inhibitory effect of nicotine over proliferation [56].

Finally, diabetes mellitus (DM) is considered the major risk factor for development of periodontitis [57]. In these patients, diabetes could alter the local environment within the periodontal pocket, favouring the growth of certain oral pathogens [57]. The lack of association between diabetes and the presence of $T$. tenax, could be influenced by our low sample size for diabetic patients with periodontal disease, so we suggest that subsequent case/control studies are conducted at a population level for both variables. However, our data agree with others, since non-significant association between type $2 \mathrm{DM}$ in patients with periodontitis and presence of oral pathogens, such as $A$. actinomycetemcomitans, $P$. gingivalis and Fusarium nucleatum, has been reported [58]; and also, non-association has been showed between diabetes and presence of T. tenax. [59]

\section{Conclusion}

We used PCR to detect T. tenax and revealed that the protozoan was frequently present in patients with periodontal disease, with an increased frequency of $T$. tenax in patients with periodontitis rather than in dental plaque-induced gingivitis. In addition, we demonstrated an association between periodontal disease development and T. tenax presence, and for first time demonstrated an association between the PSR index and T. tenax presence. Thus, we recommend screening for this protozoan in patients with periodontal disease and higher PSR indexes (between 3 and 4), mainly due to the risk of infection in other locations outside the oral cavity.

\section{Additional files}

Additional file 1: Figure S1. PCR of $\beta$-tubulin gene for T. tenax detection in patients diagnosed with periodontitis. $\beta$-tubulin PCR products of periodontitis patients (samples 1 to 30 ) were separated by electrophoresis in 1\% agarose gel and stained with Ethidium Bromide (EtBr). M: 100-bp molecular ladder marker. Pos: T. tenax strain Hs-4:NIH genomic DNA. Neg: water. (TIF $8004 \mathrm{~kb}$ )

Additional file 2: Figure S2. PCR of $\beta$-tubulin gene for T. tenax detection in patients with dental plaque-induced gingivitis. $\beta$-tubulin PCR products of gingivitis patients (samples 1 to 20) were separated by electrophoresis in 1\% agarose gel and stained with Ethidium Bromide (EtBr). M: 100-bp molecular ladder marker. Pos: T. tenax strain $\mathrm{Hs}-4: \mathrm{NIH}$ genomic DNA. Neg: water. (TIF $7791 \mathrm{~kb}$ )

\section{Abbreviations}

AAP: American Academy of Periodontology; DM: Diabetes mellitus;

DPIG: Dental plaque-induced gingivitis; EtBr: Ethidium bromide; Gl: Gingival index; PCR: Polymerase chain reaction; PSR: Periodontal Screening and Recording index; WHO: World Health Organization

\section{Acknowledgements}

We greatly appreciate the help and support in language editing manuscript to Dr. Chris Arrod.

\section{Authors' contributions}

$J \mathrm{~A}, \mathrm{PO}$ and $\mathrm{AC}$ conceived and designed the experiments. CB-W, PO, DH and $C M$. performed the experiments of amplification. CB-W, JA, and AC analysed the data. HV performed the statistical analyses and data interpretation. JB and CB-W analysed and interpreted the patient data regarding diagnosis of periodontal disease. CB-W and AC, were the major contributor in writing the manuscript. AC JG, and JA, corrected and edited the manuscript. All authors read and approved the final manuscript.

\section{Funding}

The present study was funded by the Semillero UA \# 5301 project, from the Vice-Rectorate of Research, Innovation and Postgraduate Studies of the University of Antofagasta (VRIIP-UA) and Associative Research Program (PIA) \#FB/ 001 project from Center of Biotechnology and Bio-Engineering (CeBiB). The methods indicated in this manuscript are in the patent process (Patent Pending: US $62 / 668,987)$. The funding agencies were not involved in the design of the study; collection, analysis, and interpretation of data; or in writing the manuscript.

\section{Availability of data and materials}

The data analysed during this study are included in this published paper and Additional file 1. The dataset used is available from the corresponding author on reasonable request.

\section{Ethics approval and consent to participate}

This study was approved by the Ethics Committee in Scientific Research of the University of Antofagasta (CEIC-UA); approval CEIC-REV/2014. Written informed consent to participate was obtained from all study participants.

Consent for publication

Not applicable.

\section{Competing interests}

The authors declare that they have no competing interests.

\section{Author details}

'Laboratory of Molecular Parasitology, Department of Medical Technology, Faculty of Health Sciences, University of Antofagasta, Angamos Avenue 601, P.O. Box 170, Antofagasta, Chile. ${ }^{2}$ Biomedical Departmen, Faculty of Health Sciences, University of Antofagasta, Angamos Avenue 601, P.O. Box 170, Antofagasta, Chile. ${ }^{3}$ Department of Dentistry, Faculty of Medicine and Dentistry, University of Antofagasta, Angamos Avenue 601, P.O. Box 170, Antofagasta, Chile. ${ }^{4}$ Department of Mathematics, Faculty of Basic Sciences, Universidad de Antofagasta, Angamos Avenue 601, P.O. Box 170, Antofagasta, Chile.

Received: 16 May 2018 Accepted: 13 August 2019

Published online: 04 September 2019

References

1. Honigberg BM, Lee JJ. Structure and division of trichomonas tenax (O.F. Muller). Am J Hyg. 1959;69(3):177-201.

2. Wantland WW, Lauer D. Correlation of some oral hygiene variables with age, sex, and incidence of oral protozoa. J Dent Res. 1970;49(2):293-7.

3. Masur $\mathrm{H}$, Hook E 3rd, Armstrong D. A trichomonas species in a mixed microbial meningitis. JAMA. 1976;236(17):1978-9.

4. Duboucher C, Mogenet M, Perie G. Salivary trichomoniasis. A case report of infestation of a submaxillary gland by trichomonas tenax. Arch Pathol Lab Med. 1995;119(3):277-9.

5. El Kamel A, Rouetbi N, Chakroun M, Battikh M. Pulmonary eosinophilia due to trichomonas tenax. Thorax. 1996;51(5):554-5. 
6. Krvavac S. Trichomoniasis of the breast diseased by fibrocystic mastopathy pathogenic rather than saprophytic relationship (trichomonas in fibrocystic mastopathy process). Med Arh. 1998;52(3):143-5.

7. Duboucher C, Farto-Bensasson F, Cheron M, Peltier JY, Beaufils F, Perie G. Lymph node infection by trichomonas tenax: report of a case with coinfection by mycobacterium tuberculosis. Hum Pathol. 2000;31(10):1317-21.

8. Mahmoud MS, Rahman GA. Pulmonary trichomoniasis: improved diagnosis by using polymerase chain reaction targeting trichomonas tenax 18S rRNA gene in sputum specimens. J Egypt Soc Parasitol. 2004;34(1):197-211.

9. Bellanger AP, Cabaret O, Costa JM, Foulet F, Bretagne S, Botterel F. Two unusual occurrences of trichomoniasis: rapid species identification by PCR. J Clin Microbiol. 2008;46(9):3159-61.

10. Govro EJ, Stuart MK. Cytokine response of human THP-1 macrophages to Trichomonas tenax. Exp Parasitol. 2016;169:77-80.

11. Marty M, Lemaitre M, Kemoun P, Morrier JJ, Monsarrat P. Trichomonas tenax and periodontal diseases: a concise review. Parasitology. 2017; 144(11):1417-25.

12. Menicanin D, Hynes K, Han J, Gronthos S, Bartold PM. Cementum and periodontal ligament regeneration. Adv Exp Med Biol. 2015;881:207-36.

13. Mark Welch JL, Rossetti BJ, Rieken CW, Dewhirst FE, Borisy GG. Biogeography of a human oral microbiome at the micron scale. Proc Natl Acad Sci U S A. 2016;113(6):E791-800.

14. Loe $H$, Theilade $E$, Jensen SB. Experimental gingivitis in man. J Periodontol. 1965;36:177-87

15. Kinane DF. Causation and pathogenesis of periodontal disease. Periodontol. 2001;25:8-20.

16. Kinane DF, Stathopoulou PG, Papapanou PN. Periodontal diseases. Nat Rev Dis Primers. 2017:3:17038.

17. Athari A, Soghandi L, Haghighi A, Kazemi B. Prevalence of oral Trichomoniasis in patients with periodontitis and gingivitis using PCR and direct smear. Iran J Public Health. 2007;36:33-7.

18. Kurnatowska AJ, Dudko A, Turkowicz M. Familial infections with Trichomonas tenax (O.F. Muller, 1773), Dobel, 1939. Wiad Parazytol. 2004; 50(1):35-40.

19. Mehr AK, Zarandi A, Anush K. Prevalence of Oral Trichomonas tenax in periodontal lesions of Down syndrome in Tabriz, Iran. J Clin Diagn Res. 2015;9(7):ZC88-90.

20. Turkowicz M, Tomaszewska D, Cielecka D. Molecular diagnosis of oral cavity trichomonas infections in HIV patients. Wiad Parazytol. 2004;50(2):181-6.

21. Franjola R, Puga S, Matamala F. Preliminary investigation in Trichomonas tenax in the city of Valdivia, Chile (author's transl). Bol Chil Parasitol. 1978; 33(1-2):37-8.

22. Wiebe CB, Putnins EE. The periodontal disease classification system of the American Academy of periodontology--an update. J Can Dent Assoc. 2000; 66(11):594-7.

23. Caton JG, Armitage G, Berglundh T, Chapple ILC, Jepsen S, Kornman KS, et al. A new classification scheme for periodontal and peri-implant diseases and conditions - introduction and key changes from the 1999 classification. J Periodontol. 2018;89(Suppl 1):S1-8.

24. Organization WEGoEaMfOCaWH. In: Unit OH, editor. The periodontal probe for use with the community peridontal index of treatment needs (CPITN). Geneva: World Health Organization; 1990.

25. Periodontal Screening and Recording: An early detection system [training kit]. American Dental Association and the American Academy of Periodontology (U.S.A), june 1992. Sponsored by Procter and Gamble.

26. Loe $H$. The gingival index, the plaque index and the retention index systems. J Periodontol. 1967;38(6Suppl):610-6.

27. Sambrook J, Fritis EF, Maniatis T. Molecular Cloning. A Laboratory Manual. 2 ed edn. New York: Cold Spring Harbor Laboratory Press; 1989.

28. Carlton JM, Hirt RP, Silva JC, Delcher AL, Schatz M, Zhao Q, et al. Draft genome sequence of the sexually transmitted pathogen Trichomonas vaginalis. Science. 2007;315(5809):207-12.

29. Darveau RP. Periodontitis: a polymicrobial disruption of host homeostasis. Nat Rev Microbiol. 2010;8(7):481-90.

30. Ferrara A, Conca R, Grassi L Jr, de Carneri I. Possible pathogenic role of Trichomonas tenax in chronic periodontitis. Ann Ist Super Sanita. 1986; 22(1):253-5

31. Baker JL, Bor B, Agnello M, Shi W, He X. Ecology of the Oral microbiome: beyond bacteria. Trends Microbiol. 2017;25(5):362-74.

32. Ribeiro LC, Santos C, Benchimol M. Is Trichomonas tenax a parasite or a commensal? Protist. 2015;166(2):196-210.
33. Matto J, Saarela M, Alaluusua S, Oja V, Jousimies-Somer H, Asikainen S. Detection of Porphyromonas gingivalis from saliva by PCR by using a simple sample-processing method. J Clin Microbiol. 1998;36(1):157-60.

34. Marin MJ, Ambrosio N, Herrera D, Sanz M, Figuero E. Validation of a multiplex qPCR assay for the identification and quantification of Aggregatibacter actinomycetemcomitans and Porphyromonas gingivalis: in vitro and subgingival plaque samples. Arch Oral Biol. 2018;88:47-53.

35. Bracamonte-Wolf C, Herrera D, Orrego PR, Bravo J, Araya JE. Prevalencia de Trichomonas tenax en pacientes con periodontitis crónica generalizada severa utilizando PCR convencional. In: XXVI Reunión anual de IADR, División Chile; Santiago de Chile; 2014.

36. Haubek D, Ennibi OK, Poulsen K, Vaeth M, Poulsen S, Kilian M. Risk of aggressive periodontitis in adolescent carriers of the JP2 clone of Aggregatibacter (Actinobacillus) actinomycetemcomitans in Morocco: a prospective longitudinal cohort study. Lancet. 2008:371(9608):237-42.

37. Kikuta N, Yamamoto A, Fukura K, Goto N. Specific and sensitive detection of Trichomonas tenax by the polymerase chain reaction. Lett Appl Microbiol. 1997;24(3):193-7.

38. Al-hamiary AK, Kezar Y, Al-Khafaji YA. Prevalence of oral protozoa in periodontitis and gingivitis patients whose attended to clinics periodontics, dentistry college/Babylon University. J Kufa Univ Biol. 2011:3:1-7.

39. de Albuquerque RLCJ, de Melo CM, de Santana WA, Ribeiro JL, Silva FA. Incidence of Entamoeba gingivalis and Trichomonas tenax in samples of dental biofilm and saliva from patients with periodontal disease. Revista Gaúcha de Odontología. 2011;59:35-40.

40. Khalid A, Abdulkareem EA, Kheir E, Aljafari A. Commensal oral protozoa among patients attending academic dental teaching hospital, Khartoum state, Sudan. Aljouf University Med J. 2015;2:17-20.

41. Sumaiah I, Rasha A. Evaluation of Entamoeba gingivalis and Trichomonas tenax in patients with periodontitis and gingivitis and its correlation with some risk factors. J Baghdad College Dentist. 2012;24:158-62.

42. Landry RG, Jean M. Periodontal screening and recording (PSR) index: precursors, utility and limitations in a clinical setting. Int Dent J. 2002;52(1):35-40.

43. Palmieri JR, Halverson BA, Sudjadi ST, Purnomo MS. Parasites found in the mouths of inhabitants of three villages of South Kalimantan (Borneo), Indonesia. Trop Geogr Med. 1984;36(1):57-9.

44. Vrablic J, Tomova S, Catar G. Occurrence of the protozoa, Entamoeba gingivalis and Trichomonas tenax in the mouths of children and adolescents with hyperplastic gingivitis caused by phenytoin. Bratisl Lek Listy. 1992;93(3):136-40.

45. Cambon M, Petavy AF, Guillot J, Glandier I, Deguillaume J, Coulet M. A study of the frequency of protozoa and yeasts isolated from the parodontium of 509 subjects (author's transl). Pathol Biol (Paris). 1979; 27(10):603-6.

46. Kurnatowska A, Kurnatowska A. Difficulties in the diagnosis of trichomonas infection complicated by mycosis of the oral cavity. Wiad Parazytol. 1990; 36(5-6):237-43.

47. Pardi G, Perrone M. Mazzali de llja R. Incidencia de Trichomonas tenax en pacientes con periodontitis marginal Crónica. Acta Odontol Venez. 2002:40(2):152-9.

48. Fuentes R, de la Barquera MA S, Castillo C, Hernánez-Sierra F. Prevalencia y asociación epidemiológica de los protozoarios orales Entamoeba gingivalis y Trichomonas tenax en niños mexicanos. Rev ADM. 2008;65(5):259-62.

49. Feki A, Molet B. Importance of Trichomonas tenax and Entamoebo gingivalis protozoa in the human oral cavity. Rev Odontostomatol (Paris). 1990;19(1):37-45.

50. Šegović S, Granić J, Buntak-Kobler D, Potočki-Tukša K. Trichomonas tenax in human oral cavity. Acta Stomatol Croat. 1993;27:255-61.

51. Johnson GK, Payne JB, Fili JM, Reinhardt RA, Organ CC, Slager SL. Development of smokeless tobacco-induced oral mucosal lesions. J Oral Pathol Med. 1998;27(8):388-94.

52. Kardachi BJ, Clarke NG. Aetiology of acute necrotising ulcerative gingivitis: a hypothetical explanation. J Periodontol. 1974;45(11):830-2.

53. Mirbood S, Ahing S, Pruthi V. Immunohistochemical study of vestibular blood vessel density and internal circumference in smokers and non smokers. J Pak Med Assoc. 1994;44:210-2.

54. Lehker MW, Arroyo R, Alderete JF. The regulation by iron of the synthesis of adhesins and cytoadherence levels in the protozoan trichomonas vaginalis. J Exp Med. 1991;174(2):311-8.

55. Shivji M, Burger S, Moncada CA, Clarkson AB Jr, Merali S. Effect of nicotine on lung S-adenosylmethionine and development of pneumocystis pneumonia. J Biol Chem. 2005;280(15):15219-28. 
56. Baek O, Zhu W, Kim HC, Lee SW. Effects of nicotine on the growth and protein expression of Porphyromonas gingivalis. J Microbiol. 2012;50(1):143-8.

57. Preshaw PM, Alba AL, Herrera D, Jepsen S, Konstantinidis A, Makrilakis

$\mathrm{K}$, et al. Periodontitis and diabetes: a two-way relationship. Diabetologia. 2012;55(1):21-31.

58. Field CA, Gidley MD, Preshaw PM, Jakubovics N. Investigation and quantification of key periodontal pathogens in patients with type 2 diabetes. J Periodontal Res. 2012:47(4):470-8.

59. Nocito-Mendoza I, Vaconi-Correas MD, Ponce de Leon-Horianski P, ZderoPandzich M. Entamoeba gingivalis y trichomonas tenax en pacientes diabéticos. RCOE. 2003:8(1):13-23.

\section{Publisher's Note}

Springer Nature remains neutral with regard to jurisdictional claims in published maps and institutional affiliations.

Ready to submit your research? Choose BMC and benefit from:

- fast, convenient online submission

- thorough peer review by experienced researchers in your field

- rapid publication on acceptance

- support for research data, including large and complex data types

- gold Open Access which fosters wider collaboration and increased citations

- maximum visibility for your research: over $100 \mathrm{M}$ website views per year

At BMC, research is always in progress.

Learn more biomedcentral.com/submissions 